\title{
The Impact of SARS-CoV-2 Infection on Pregnancy
}

\begin{abstract}
Pregnant women are considered a vulnerable group due to the unique immunological changes that take place during pregnancy ${ }^{2}$. It is important to understand the impact of SARS-CoV-2 on pregnant women and foetuses, in order to produce clinical guidelines for obstetric and neonatal management. In recent months, there has been arise in research into the impact of SARS-CoV-2 infection on pregnancy.
\end{abstract}

This essay aims to explore this emerging data and understand the impact of SARS-CoV-2 on pregnancy, including looking at specific subpopulations, such as women from Black and Minority Ethnic (BAME) backgrounds. In addition, it will explore the data surrounding whether vertical transmission from mother to baby is possible. This is an area of considerable controversy.

Keywords SARS-CoV-2, pregnancy, BAME

\section{Introduction}

First reported in Wuhan, China in December 2019, the novel coronavirus (severe acute respiratory syndrome coronavirus 2 [SARS-CoV2] which causes coronavirus disease 2019 [COVID-19]) has spread rapidly throughout the world. It has become a global public health crisis, and was declared a pandemic on $11^{\text {th }}$ March 2020 by the World Health Organisation ${ }^{1}$.

Pregnant women are considered a vulnerable group due to the unique immunological changes that take place during pregnancy ${ }^{2}$. It is important to understand the impact of SARS-CoV-2 on pregnant women and foetuses, in order to produce clinical guidelines for obstetric and neonatal management. In recent months, there has been arise in research into the impact of SARS-CoV-2 infection on pregnancy.

This essay aims to explore this emerging data and understand the impact of SARS-CoV-2 on pregnancy, including looking at specific subpopulations, such as women from Black and
Triya Chakravorty BA (Oxon)

University of Oxford, UK

Triya.chakravorty@queens.oxon.ac.uk

Cite as: Chakravorty, T. (2020) The impact of SARS-Cov-2 infection on pregnancy. Sushruta J Health Policy \& Opin vol 14; issue 1: epub 22.11.2020 DOI: https://doi.org/10.38192/14.1.2

Article Information

Submitted $\quad 08.11 .2020$

Epub 22.11.2020

Creative commons licence v4.0

Minority Ethnic (BAME) backgrounds. In addition, it will explore the data surrounding whether vertical transmission from mother to baby is possible. This is an area of considerable controversy.

\section{Pregnancy as a Risk Factor for Viral Infection}

The immunological changes that occur during pregnancy are complex and have been the focus of research for many years. There are concerns as to whether pregnant women are more susceptible to viral infection, and how infection impacts maternal health, pregnancy outcomes and foetal health. There are several implicated factors, including the type of microorganism involved and the stage of pregnancy that infection occurs ${ }^{2}$.

In previous viral pandemics, pregnant women have been considered a high-risk population group for severe illness and in some cases, such as with influenza, have been prioritised for vaccination $^{3}$. Murine studies of influenza have shown that pregnancy increases influenza- 
related pathology via mechanisms such as impaired viral clearance, increased levels of proinflammatory cytokines and hormone induced increased physiological lung stress ${ }^{4}$.

A meta-analysis conducted in 2019 showed that influenza infection in pregnancy was associated with a seven times increased risk of hospitalisation, although multivariable analysis showed no change in mortality or rate of admission to an intensive care unit (ICU), when adjusting for risk factors such as age and comorbidities $^{3}$.

When considering the impact of SARS-CoV-2 infection on pregnant women, it is useful to look at the data from previous coronavirus outbreaks regarding pregnancy, although this is relatively limited. Case studies from previous coronavirus outbreaks (e.g. Severe Acute Respiratory Syndrome [SARS] and Middle East Respiratory Syndrome [MERS]) have suggested that infection during pregnancy is associated with adverse maternal outcomes, including higher mortality and admission to ICU, as well as adverse fetal outcomes, including preterm delivery and intrauterine growth restriction ${ }^{5,6}$. In a case study of 12 pregnant women infected with SARS-CoV in 2003 , the mortality rate was $25 \% 5$, compared to the average global mortality of $10 \%{ }^{7}$.

\section{Impact of COVID-19 on Pregnant Women}

SARS-CoV-2 infection during pregnancy is associated with adverse maternal outcomes, including increased risk of caesarean delivery, need for invasive ventilation and higher rate of admission to an ICU. It is also associated with adverse fetal outcomes, including preterm delivery, intrauterine growth restriction and increased risk of admission to a neonatal intensive care unit (NICU) ${ }^{8-10}$. There is emerging evidence, however retrospective design, inconsistent definition and reporting of outcomes and lack of availability of universal testing for SARS-CoV-2 infection, makes analysing the data on a larger scale, more difficult. However, these inconsistencies are understandable given the recent nature of the SARS-CoV-2 outbreak, as well as the ever-changing classification of signs and symptoms associated with infection.

Khalil et al. ${ }^{9}$ carried out a systematic review and meta-analysis of 86 studies investigating SARS$\mathrm{CoV}-2$ infection and pregnancy, making it the largest meta-analysis of its kind to date. The results showed that pregnant women with SARS-
CoV-2 infection were at an increased risk of iatrogenic preterm birth and caesarean delivery. Preterm birth was seen in around one in five cases, and $50 \%$ of births were carried out by caesarean section. Although few studies reported the indications for caesarean delivery, the most common reported indications were due to complications of SARS-CoV-2 infection, including severe maternal pneumonia and risk of sudden maternal decompensation. The meta-analysis results regarding mortality were reassuring, as maternal mortality was found to be similar to that of non-pregnant women of reproductive age ${ }^{9}$.

Similar findings were seen in the Centre for Disease Control and Prevention (CDC) Morbidity and Mortality Weekly Report in June 2020, which analysed data from over 300,000 women in the United States of America (USA) of reproductive age (defined as between 15-44 years) ${ }^{10}$. In their cohort, SARS-CoV-2 infection during pregnancy was associated with greater risk of hospitalisation, admission to ICU and mechanical ventilation. They also did not find an increase in maternal mortality.

There is also emerging evidence to suggest that increased maternal age, high body mass index and pre-existing comorbidities are associated with more severe disease ${ }^{8}$. These reflect the risk factors seen in the general population as well.

\section{Clinical Manifestations of SARS-CoV-2 Infection} in Pregnancy

There is evidence to suggest that the clinical symptoms of SARS-CoV-2 infection are different in pregnant women. For example, a systematic review and meta-analysis published by Allotey et al. ${ }^{8}$ of 77 studies involving pregnant women with SARS-CoV-2 infection, showed that although the most common clinical manifestations of SARSCoV-2 infection in pregnancy were fever and cough, pregnant women were less likely to report symptoms of fever and myalgia, when compared with non-pregnant women of reproductive age. Furthermore, the CDC report showed that the percentages of infected pregnant women reporting fever, muscle aches, chills, headache and diarrhoea were lower than those reported in the non-pregnant group ${ }^{10}$.

These results suggest that the clinical symptoms of SARS-CoV-2 infection may be different in pregnant women. There may also be higher rates of asymptomatic infection in pregnant women compared to non-pregnant women ${ }^{8}$. This is 
significant, given that the lack of a universal and reliable test for SARS-CoV-2 means that clinicians rely on signs and symptoms for clinical diagnosis. This also has implications for the reliability of data based on a clinical diagnosis of infection.

These findings are enough to warrant the need for universal SARS-CoV-2 testing of pregnant women, regardless of symptoms, in order to ensure adequate monitoring for clinical deterioration is in place, staff are protected from infection and neonates are managed expectantly. The importance of universal testing of pregnant women was shown in a case study from the USA, which found that in a cohort of 215 pregnant women who were tested regardless of symptoms when admitted for delivery at two centres, $90 \%$ of those who tested positive for SARS-CoV-2 had no symptoms at admission ${ }^{11}$.

\section{Impact of Ethnicity on Pregnancy Outcomes}

There is emerging evidence that people from Black and Minority Ethnic (BAME) groups in the UK are disproportionately impacted by SARSCoV-2 infection, with an increased risk of admission to ICU and death ${ }^{12}$. Given that it is well established that BAME women in the UK have disproportionately higher rates of adverse outcomes and maternal mortality compared to white women ${ }^{13}$, it is important to consider whether BAME pregnant women may represent a sub-group of pregnant women who are at a greater risk of adverse outcomes from SARS-CoV2 infection. Furthermore, it is likely that the short- and long-term consequences of the COVID19 pandemic will only worsen pre-existing healthcare inequalities.

The meta-analysis carried out by Khalil et al. ${ }^{9}$ showed that the prevalence of BAME background and maternal co-morbidities were high in pregnant women infected with SARS-CoV-2. Furthermore, the CDC report showed that in the USA, Hispanic and non-Hispanic black pregnant women were disproportionately affected by SARS-CoV-2 infection during pregnancy, compared to their white peers ${ }^{10}$. This was apparent despite the fact that data regarding ethnicity was missing from $20 \%$ of women in their cohort.

In a prospective cohort study using the UK Obstetric Surveillance System, Knight et al.14 found that BAME pregnant women had a higher rate of hospital admission compared to their white peers. They found that out of the 427 pregnant women admitted to hospital with confirmed SARS-CoV-2 infection between $1^{\text {st }}$ March and 14th April 2020, 69\% were from BAME backgrounds. This over-representation of BAME individuals could be due to a higher risk of infection, a higher risk of severe disease, or both. There are several proposed reasons for the differences in outcomes for BAME women, including co-morbidities, social and health behaviours ${ }^{15}$. The reasoning is likely to be multifactorial, and further investigation is warranted.

\section{The Possibility of Vertical Transmission}

Whether vertical transmission of SARS-CoV-2 from mother to baby is possible is a topic of much controversy. There are some theories about how vertical transmission may occur, but no concrete proof exists to date. The angiotensin-converting enzyme 2 (ACE2) receptor is now considered to be the cell surface receptor for the SARS-CoV-2 virus ${ }^{16}$. ACE2 receptors have been shown to be expressed in the human placenta ${ }^{17}$, and a rat model showed that ACE2 receptor expression increases during pregnancy in the uterus and placenta ${ }^{18}$. Therefore, it is theorised that the virus may be able to spread transplacentally via the ACE2 receptor.

The practical approach to investigating whether vertical transmission occurs involves testing for the presence of SARS-CoV-2 virus in placental fluid, amniotic fluid, cord-blood and neonatal pharyngeal swab samples ${ }^{19}$. However, strict time and infection control measures are needed to ensure that the samples taken from the neonate represent the intrauterine conditions. The samples need to be collected immediately after delivery and using an aseptic technique.

The first study investigating the possibility of vertical transmission came from Zhongnan Hospital in Wuhan. There, Chen et al. ${ }^{20}$ conducted a retrospective analysis of nine pregnant women with mild to moderate COVID-19 pneumonia contracted in the third trimester. In this cohort, they found no evidence of vertical transmission, which was assessed by testing for the presence of SARS-CoV-2 in amniotic fluid, cord blood and neonatal throat swab samples using quantitative reverse transcriptase polymerase chain reaction (qRT-PCR).

However, other case studies suggest that vertical transmission may be possible. For example, Zeng et al. ${ }^{21}$ conducted a cohort study involving 33 neonates born to mothers with SARS-CoV-2 
infection in Wuhan Children's Hospital. In this cohort, three neonates $(9 \%)$ tested positive for SARS-CoV2-infection and showed symptoms of viral illness. These neonates were born via caesarean section under strict infection control measures. Based on this, the authors concluded that the neonates likely were infected in utero. However, it must be noted that the first neonatal diagnostic test was not carried out until the second day of life, which means that the possibility that infection occurred after birth cannot be ruled out.

More recently, there have been studies investigating the possibility of vertical transmission by testing for SARS-CoV-2-specific antibodies (immunoglobulin G [IgG] and immunoglobulin $\mathrm{M}$ [IgM]) in neonatal samples using automated chemiluminescence immunoassays ${ }^{22,23}$. Dong et al. ${ }^{22}$ reported that a neonate born to a mother with SARS-CoV-2 infection in Renmin Hospital, Wuhan had elevated anti-SARS-CoV-2 IgM and IgG antibody levels two hours after birth. The elevated levels of IgM antibodies suggest that the neonate was infected in-utero, as IgM antibodies do not cross the placenta, and therefore may represent a neonatal immune response to in-utero infection ${ }^{24}$. The precautionary measures taken at the time of birth (including caesarean delivery in a negative-pressure isolation room, mother wearing an N95 respirator mask, no motherneonate contact and immediate quarantine of the neonate) decrease the likelihood that infection occurred during or after delivery, however the possibility cannot be ruled out 22 .

Anti-SARS-CoV-2 IgM antibodies were also obtained in blood samples from two neonates in another case report ${ }^{23}$. However, for the three neonates across these two studies, pharyngeal swab samples were negative for SARS-CoV-2 RNA and cord-blood and placental sampling were not performed, indicating that direct evidence of infection was absent. Therefore, these case studies should only be considered preliminary observations which warrant further examination of maternal and neonatal samples.

It is important to note that the majority of case studies reporting about vertical transmission include women who acquired SARS-CoV-2 infection in the third trimester of pregnancy. There remains little data on perinatal outcomes when the infection is acquired in early pregnancy. Given that it is theorised that pregnancy has three distinct immunological phases, each characterised by distinct physiological processes, it is important to consider that infection acquired earlier might have different impacts on the mother and fetus ${ }^{2}$.

Furthermore, in infection acquired in the third trimester, the time between clinical manifestation of the infection in the mother and delivery is relatively short. It is thought that in some viral infections, the placental barrier may temporarily delay the transfer of virus from mother to foetus. Therefore, it is possible that there might be a risk of vertical transmission when infection occurs in the first or second trimester, where there is a longer interval between clinical manifestation and delivery ${ }^{19}$.

Further research is required to investigate the possibility of vertical transmission. Ideally, this should involve universal collection of a range biological samples (including cord-blood, placental tissue, maternal vaginal swabs and neonatal nasopharyngeal swabs) from infected mothers and neonates immediately post-delivery using an aseptic technique. Furthermore, investigation into the risks of infection in early pregnancy is needed, specifically looking at the risk of adverse fetal outcomes such as structural malformations and fetal growth restriction. In the UK, the ongoing "periCOVID" trial aims to investigate the risk of COVID-19 infection in neonates born to infected mothers and to determine the potential routes of vertical transmission ${ }^{25}$.

\section{Conclusion}

The evidence suggests that pregnant women with SARS-CoV-2 infection are at an increased risk of some adverse maternal and fetal outcomes. Reassuringly, however, there seems to be no negative impact on maternal mortality. Whilst vertical transmission of SARS-CoV-2 infection from mother to baby may be possible, due to the limited and conflicting data currently available, it is not possible yet to draw definitive conclusions. The collection of longitudinal data will be required to fully understand the impact of SARSCoV-2 infection on maternal and neonatal outcomes. Going forward, it will be important to include pregnant women in SARS-CoV-2 vaccine trials, as when a vaccine does become widely available, pregnant women may need to be classed as a priority group.

\section{References:}


World Health Organisation Coronavirus disease (COVID-19) pandemic,

$<$ https://www.euro.who.int/en/healthtopics/health-emergencies/coronaviruscovid-19> (2020).

2 Mor, G. \& Cardenas, I. The immune system in pregnancy: a unique complexity. Am J Reprod Immunol 63, 425-433, doi:10.1111/j.16000897.2010.00836.x (2010).

Mertz, D. et al. Pregnancy as a risk factor for severe influenza infection: an individual participant data metaanalysis. BMC Infect Dis 19, 683, doi:10.1186/s12879-019-4318-3 (2019).

$4 \quad$ Littauer, E. Q. et al. H1N1 influenza virus infection results in adverse pregnancy outcomes by disrupting tissue-specific hormonal regulation. PLOS Pathogens 13, e1006757, doi:10.1371/journal.ppat.1006757 (2017). Wong, S. F. et al. Pregnancy and perinatal outcomes of women with severe acute respiratory syndrome. Am J Obstet Gynecol 191, 292-297, doi:10.1016/j.ajog.2003.11.019 (2004). Lam, C. M. et al. A case-controlled study comparing clinical course and outcomes of pregnant and non-pregnant women with severe acute respiratory syndrome. BJOG: An International Journal of Obstetrics \& Gynaecology 111, 771-774, doi:10.1111/j.1471-0528.2004.00199.x (2004).

Who. (World Health Organization, 2003).

Allotey, J. et al. Clinical manifestations, risk factors, and maternal and perinatal outcomes of coronavirus disease 2019 in pregnancy: living systematic review and meta-analysis. BMJ 370, m3320, doi:10.1136/bmj.m3320 (2020). Khalil, A. et al. SARS-CoV-2 infection in pregnancy: A systematic review and meta-analysis of clinical features and pregnancy outcomes. EClinicalMedicine 25, doi:10.1016/j.eclinm.2020.100446 (2020).

10 Ellington S, S. P., Tong VT, et al. Characteristics of Women of Reproductive Age with LaboratoryConfirmed SARS-CoV-2 Infection by Pregnancy Status - United States,
January 22-June 7, 2020. . MMWR Morb Mortal Wkly Rep doi:http://dx.doi.org/10.15585/mmwr. mm6925a1 (2020).

11 Sutton, D., Fuchs, K., D'Alton, M. \& Goffman, D. Universal Screening for SARS-CoV-2 in Women Admitted for Delivery. New England Journal of Medicine 382, 2163-2164, doi:10.1056/NEJMc2009316 (2020). Public Health England. Disparities in the risk and outcomes of covid-19. (2020).

Garcia, R., Ali, N., Papadopoulos, C. \& Randhawa, G. Specific antenatal interventions for Black, Asian and Minority Ethnic (BAME) pregnant women at high risk of poor birth outcomes in the United Kingdom: a scoping review. BMC Pregnancy and Childbirth 15, 226, doi:10.1186/s12884015-0657-2 (2015).

14 Knight, M. et al. Characteristics and outcomes of pregnant women admitted to hospital with confirmed SARS-CoV-2 infection in UK: national population based cohort study. BMJ 369, m2107, doi:10.1136/bmj.m2107 (2020).

15 Pareek, M. et al. Ethnicity and COVID-19: an urgent public health research priority. Lancet 395, 1421-1422, doi:10.1016/S0140-6736(20)30922-3 (2020).

Zhao, Y. et al. Single-cell RNA expression profiling of ACE2, the receptor of SARSCoV-2. bioRxiv, 2020.2001.2026.919985, doi:10.1101/2020.01.26.919985 (2020). Valdes, G. et al. Distribution of angiotensin-(1-7) and ACE2 in human placentas of normal and pathological pregnancies. Placenta 27, 200-207, doi:10.1016/j.placenta.2005.02.015 (2006).

18 Levy, A. et al. ACE2 expression and activity are enhanced during pregnancy. American Journal of PhysiologyRegulatory, Integrative and Comparative Physiology 295, R1953-R1961, doi:10.1152/ajpregu.90592.2008 (2008).

19 Wang, C., Zhou, Y.-H., Yang, H.-X. \& Poon, L. C. Intrauterine vertical transmission of SARS-CoV-2: what we know so far. Ultrasound in Obstetrics \& Gynecology 55, 724-725, doi:10.1002/uog.22045 (2020). 


\section{Sushruta}

20 Chen, H. et al. Clinical characteristics and intrauterine vertical transmission potential of COVID-19 infection in nine pregnant women: a retrospective review of medical records. The Lancet 395, 809815, doi:10.1016/S01406736(20)30360-3 (2020).

21 Zeng, L. et al. Neonatal Early-Onset Infection With SARS-CoV-2 in 33 Neonates Born to Mothers With COVID19 in Wuhan, China. JAMA Pediatrics, doi:10.1001/jamapediatrics.2020.0878 (2020).

22 Dong, L. et al. Possible Vertical Transmission of SARS-CoV-2 From an Infected Mother to Her Newborn. JAMA, doi:10.1001/jama.2020.4621 (2020).

23 Zeng, H. et al. Antibodies in Infants Born to Mothers With COVID-19 Pneumonia. JAMA 323, 1848-1849, doi:10.1001/jama.2020.4861 (2020).

24 Palmeira, P., Quinello, C., Silveira-Lessa, A. L., Zago, C. A. \& Carneiro-Sampaio, M. IgG placental transfer in healthy and pathological pregnancies. Clin Dev Immunol 2012, 985646, doi:10.1155/2012/985646 (2012).

25 Institute for Infection and Immunity. periCOVID - Understanding COVID-19 Infection in Women and Their Babies, $<$ https://www.pericovid.com/> (2020). 